\title{
Evaluation of the $\beta^{+}$-decay $\log f t$ value with inclusion of the neutron-proton pairing and particle-number projection
}

\author{
S. Kerrouchi ${ }^{1,2,3, a}$, N. H. Allal ${ }^{3}$, M. Fellah ${ }^{3}$, and M. R. Oudih ${ }^{3}$ \\ ${ }^{1}$ Ecole Nationale Supérieure de Technologie ENST ex CT-siège DG SNVI, RN5, Z. I. Rouiba, Algiers, Algeria \\ ${ }^{2}$ Ecole Nationale Préparatoire pour les Etudes de l'Ingénieur, RN5 Rouiba, Algiers, Algeria \\ ${ }^{3}$ Laboratoire de Physique Théorique, Faculté de Physique, USTHB, BP32 El Alia, 16111 Bab Ezzouar, Algiers, Algeria
}

\begin{abstract}
The neutron-proton isovector pairing effect on the beta-plus decay log $f t$ values is studied in typical mirror $N \simeq Z$ nuclei. The $\log f t$ values are calculated by including or not the isovector pairing before and after a particle-number projection using the Sharp-Bardeen-Cooper-Schrieffer (SBCS) method. It is shown that the values obtained after projection in the isovector pairing case are the closest ones to experimental data. The effect of the deformation of the mother and daughter nuclei on the $\log f t$ is also studied.
\end{abstract}

\section{Introduction}

Beta decay of nuclei is a key process to access to the structure of the atomic nucleus. Although these transitions are, in principle, well-understood, a satisfactory theoretical description of this process took place only in the 1990s. This is due to the complexity of the structure of the nucleus and the different residual interactions involved in this process. One of the residual interactions with a key role in the description of nuclei is the pairing interaction between nucleons $(p-p, n-n$ and $n-p)$. This interaction may exist in two different forms, isoscalar $(T=0)$ taking into account only the neutron-proton $(n-p)$ pairing or isovector $(T=1)$ which takes into account three different types of pairing (i.e., $p-p, n-n$ and $n-p$ ). In order to avoid cumbersome calculations, in both analytical and numerical sides, we take into account only the isovector pairing in the present work.

The $n-p$ pairing has a major role in the structure of $N \simeq Z$ nuclei [1] and in particular in the evaluation of the beta transition probability [2-4].

To include $n-p$ pairing, there exist many approaches. One of the most popular is the Bardeen, Cooper and Schrieffer (BCS) one [5]. However, the main shortcoming of this method is the non-conservation of the particle number. Several projection methods have thus been proposed in the $n-p$ pairing case (see, e.g., in Refs. $[6,7]$ ).

In this contribution, we propose to study the isovector $n-p$ pairing effect on the $\beta^{+}$transition probability and therefore on the value of $\log f t$ which characterizes these transitions. This is done within the framework of the BCS approach. As the BCS theory does not conserve the particle number, a particle-number projection is performed using the Sharp-BCS (SBCS) exact projection method [8]

\footnotetext{
a e-mail: kerrouchis@gmail.com
}

which has been extended to the isovector pairing case [912].

As a first step, the expressions of the transition probabilities, of Fermi as well as Gamow-Teller types, which strictly conserve the particle number are established. As a second step, they are calculated for some transitions between mirror nuclei $N \simeq Z$ within the framework of the Woods-Saxon model. The effect of the deformation of the mother and daughter nuclei on the $\log f t$ is also studied.

\section{Formalism}

\subsection{Hamiltonian}

In the second quantization and isospin formalism, a system constituted by $Z$ protons and $N$ neutrons is described, in the isovector case, by the Hamiltonian:

$$
\begin{aligned}
\hat{H}= & \sum_{v>0, t} \varepsilon_{v t}\left(a_{v t}^{+} a_{v t}+a_{\widetilde{v} t}^{+} a_{\widetilde{v} t}\right)- \\
& \frac{1}{2} \sum_{t t^{\prime}} G_{t t^{\prime}} \sum_{v, \mu>0}\left(a_{v t}^{+} a_{\widetilde{v} t^{\prime}}^{+} a_{\widetilde{\mu} t^{\prime}} a_{\mu t}+a_{v t}^{+} a_{\widetilde{v} t^{\prime}}^{+} a_{\widetilde{\mu}} a_{\mu t^{\prime}}\right),
\end{aligned}
$$

where the subscript $t$ corresponds to the isospin component $(t=n, p), a_{v t}^{+}$and $a_{v t}$, respectively, represent the creation and the annihilation operators of the particle in the state $|v t\rangle$, of energy $\varepsilon_{v t} ;|\tilde{v} t\rangle$ is the time-reversed state of $|v t\rangle . G_{t t^{\prime}}$ is the pairing strength which is assumed to be constant. Neutrons and protons are assumed to occupy the same energy levels.

\subsection{Ground state}

The ground-state is expressed in the particle representation as a tensorial product of individual wave functions $\left|\Psi_{j}\right\rangle$. It 
is then given by [9]

$$
|\psi\rangle=\prod_{j>0}\left|\Psi_{j}\right\rangle
$$

with:

$$
\begin{aligned}
\left|\Psi_{j}\right\rangle=\left[B_{1}^{j} A_{j p}^{+} A_{j n}^{+}+\right. & B_{p}^{j} A_{j p}^{+}+B_{n}^{j} A_{j n}^{+}+ \\
& \left.B_{4}^{j}\left(a_{j p}^{+} a_{j n}^{+}+a_{j n}^{+} a_{j p}^{+}\right)+B_{5}^{j}\right]|0\rangle .
\end{aligned}
$$

The coefficients $B_{i}^{j}(i=1, p, n, 4,5)$ being the occupation probability amplitudes of the $j$ state and $A_{j t}^{+}=a_{j t}^{+} a_{j t}^{+}, t=n, p$. However, this state could only describe even systems but not odd ones. For an odd system let us denote $|\pi\rangle$ the wavefunction of an odd $(Z)$-even $(N)$ nucleus, and $|v\rangle$ that of an even $(Z)$-odd $(N)$ nucleus. If one assumes that the blocked level is $\mu$, they are given by

$$
|\pi\rangle=a_{\mu p}^{+} \prod_{\substack{j>0 \\ j \neq \mu}}\left|\Psi_{j}\right\rangle,|v\rangle=a_{\mu n}^{+} \prod_{\substack{j>0 \\ j \neq \mu}}\left|\Psi_{j}\right\rangle .
$$

Let us note here that in expressions (4), the coefficients $B_{i}^{j}$ depend on $\mu$. In order to simplify the notations, this dependence is omitted in what follows.

However, it is well known that the BCS states are not eigenstates of the particle number operator. The wave functions (4) must then be projected. In the present work, we use a projection method derived from the Sharp-BCS one [8], which is discrete. The projected homologue of the state $|\pi\rangle$ is given by

$$
\begin{array}{r}
\left|\pi_{m m^{\prime}}\right\rangle=C_{m m^{\prime}}^{\pi}\left[\sum_{k=1}^{m} \sum_{k^{\prime}=1}^{m^{\prime}} z_{k}^{-N} z_{k^{\prime}}^{-Z}\left|\pi\left(z_{k}, z_{k^{\prime}}\right)\right\rangle+\right. \\
\left.z_{k}^{-N} \bar{z}_{k^{\prime}}^{-Z}\left|\pi\left(z_{k}, \bar{z}_{k^{\prime}}\right)\right\rangle+\text { c.c. }\right],
\end{array}
$$

where

$$
\begin{aligned}
&\left|\pi\left(z_{k}, z_{k^{\prime}}\right)\right\rangle=a_{\mu p}^{+} \prod_{\substack{j>0 \\
j \neq \mu}}\left[z_{k}^{2} z_{k^{\prime}}^{2} B_{1}^{j} A_{j p}^{+} A_{j n}^{+}+\right. \\
& z_{k^{\prime}}^{2} B_{p}^{j} A_{j p}^{+}+z_{k}^{2} B_{n}^{j} A_{j n}^{+}+ \\
&\left.\quad z_{k} z_{k^{\prime}} B_{4}^{j}\left(a_{j p}^{+} a_{j n}^{+}+a_{j n}^{+} a_{j p}^{+}\right)+B_{5}^{j}\right]|0\rangle,
\end{aligned}
$$

$m$ and $m^{\prime}$, which are positive integers, are the extraction degrees of the false components, $z_{k}=\exp (i k \pi / 2 m)$, c.c means the complex conjugate with respect to $z_{k}$ and $C_{m m^{\prime}}^{\pi}$ is the normalization constant. One obtains an analogous expression for the state $\left|v_{m m^{\prime}}\right\rangle$.

\subsection{Beta decay $\log f t$ values}

It is well known that the half-lives of the beta transitions $t_{1 / 2}$ are given by $[13,14]$ :

$$
t_{1 / 2}=\frac{\ln 2}{\lambda_{i f}}, \text { where } \lambda_{i f}=\frac{\ln 2 \cdot f_{0}\left(B_{F}+B_{G T}\right)}{\kappa} .
$$

Thus,

$$
f t=\frac{\kappa}{B_{F}+B_{G T}}, \text { where } \kappa=6147 \mathrm{~s},
$$

and the Fermi $\left(B_{F}\right)$ and Gamow-Teller $\left(B_{G T}\right)$ transition probability amplitudes are given by

$$
B_{F}\left(\operatorname{resp} . B_{G T}\right)=\frac{g_{V}^{2}\left(\operatorname{resp} \cdot g_{A}^{2}\right)}{2 J_{i}+1}\left|\mathcal{M}_{F}\right|^{2}\left(\operatorname{resp} .\left|\mathcal{M}_{G T}\right|^{2}\right)
$$

The $\log f t$ value allows one to make a classification of the transition [5]. $f_{0}$ is a phase-space integral that contains the lepton kinematics; $g_{V}=1$ is the vector coupling constant of the weak interaction [5] and $g_{A}=1.25$ is the axialvector coupling constant of the weak interaction [5]. $J_{i}$ is the angular momentum of the initial nuclear state. The two matrix elements $\mathcal{M}_{F}$ and $\mathcal{M}_{G T}$ corresponding to the Fermi and Gamow-Teller transitions may be written in the second quantization and isospin formalism

$$
\begin{gathered}
\mathcal{M}_{F}=\sum_{l l^{\prime}}\left\langle l^{\prime}|\mathbf{1}| l\right\rangle\left\langle\psi^{f}\left|a_{l^{\prime} n}^{+} a_{l p}\right| \psi^{i}\right\rangle \\
\mathcal{M}_{G T}=\sum_{l l^{\prime}} \sum_{\tau}\left\langle l^{\prime}\left|\sigma_{\tau}\right| l\right\rangle\left\langle\psi^{f}\left|a_{l^{\prime} n}^{+} a_{l p}\right| \psi^{i}\right\rangle,
\end{gathered}
$$

where $\left\langle l^{\prime}|\mathbf{1}| l\right\rangle$ and $\left\langle l^{\prime}\left|\sigma_{\tau}\right| l\right\rangle$, respectively, represent the matrix elements on the single-particle basis of the unit operator 1 and the Pauli spin operator $\sigma_{\tau}(\tau=-1,0,1)$. Using Eq. (4) (i.e. before projection) $\mathcal{M}_{F}$ and $\mathcal{M}_{G T}$ are given by

$$
\begin{gathered}
\mathcal{M}_{F}=\prod_{\substack{j>0 \\
j \neq \mu}}\left[B_{1}^{j i} B_{1}^{j f}+B_{p}^{j i} B_{p}^{j f}+B_{n}^{j i} B_{n}^{j f}+\right. \\
\left.2 B_{4}^{j i} B_{4}^{j f}+B_{5}^{j i} B_{5}^{j f}\right], \\
\mathcal{M}_{G T}=\mu \prod_{\substack{j>0 \\
j \neq \mu}}\left[B_{1}^{j i} B_{1}^{j f}+B_{p}^{j i} B_{p}^{j f}+\right. \\
\left.B_{n}^{j i} B_{n}^{j f}+2 B_{4}^{j i} B_{4}^{j f}+B_{5}^{j i} B_{5}^{j f}\right] .
\end{gathered}
$$

In the same way, after projection, one has

$$
\begin{gathered}
\mathcal{M}_{F}=C_{m m^{\prime}}^{\pi} C_{m m^{\prime}}^{v}\left\{\sum_{k=1}^{m} \sum_{k^{\prime}=1}^{m^{\prime}} z_{k}^{-N} z_{k^{\prime}}^{-Z} T\left(z_{k}, z_{k^{\prime}}\right)+\right. \\
\left.z_{k}^{-N} \bar{z}_{k^{\prime}}^{-Z} T\left(z_{k}, \bar{z}_{k^{\prime}}\right)+\text { c.c. }\right\}, \\
\mathcal{M}_{G T}=C_{m m^{\prime}}^{\pi} C_{m m^{\prime}}^{v} \mu\left\{\sum_{k=1}^{m} \sum_{k^{\prime}=1}^{m^{\prime}} z_{k}^{-N} z_{k^{\prime}}^{-Z} T\left(z_{k}, z_{k^{\prime}}\right)\right. \\
\left.+z_{k}^{-N} \bar{z}_{k^{\prime}}^{-Z} T\left(z_{k}, \bar{z}_{k^{\prime}}\right)+\text { c.c }\right\},
\end{gathered}
$$

where

$$
\begin{aligned}
T\left(z_{k}, z_{k^{\prime}}\right)=\prod_{\substack{j>0 \\
j \neq \mu}}\left[z_{k}^{2} z_{k^{\prime}}^{2} B_{1}^{j i} B_{1}^{j f}+z_{k^{\prime}}^{2} B_{p}^{j i} B_{p}^{j f}+\right. \\
\left.z_{k}^{2} B_{n}^{j i} B_{n}^{j f}+2 z_{k} z_{k^{\prime}} B_{4}^{j i} B_{4}^{j f}+B_{5}^{j i} B_{5}^{j f}\right] .
\end{aligned}
$$


Table 1. Values of the ground state deformation parameters and the pairing strengths (in $\mathrm{MeV}$ ) for the nuclei considered in the present study.

\begin{tabular}{lccccc}
\hline Nucleus & $c$ & $h$ & $\Delta_{p p}$ & $\Delta_{n n}$ & $\Delta_{n p}$ \\
\hline \hline${ }^{25} \mathrm{Al}$ & 1.213 & 0.104 & 0.105 & 0.118 & 0.102 \\
\hline${ }^{25} \mathrm{Mg}$ & 1.216 & 0.078 & 0.100 & 0.118 & 0.102 \\
\hline${ }^{49} \mathrm{Mn}$ & 1.164 & -0.156 & 0.080 & 0.083 & 0.077 \\
\hline${ }^{49} \mathrm{Cr}$ & 1.164 & -0.156 & 0.078 & 0.084 & 0.078 \\
\hline${ }^{53} \mathrm{Co}$ & 1.063 & $5.57 E-3$ & 0.078 & 0.080 & 0.077 \\
\hline${ }^{53} \mathrm{Fe}$ & 1.063 & $5.57 E-3$ & 0.076 & 0.081 & 0.076 \\
\hline
\end{tabular}

Table 2. Values of the relative discrepancy (\%) between theoretical and experimental results (see the text for notations).

\begin{tabular}{ccccc}
\hline & ${ }^{25} \mathrm{Al}-{ }^{25} \mathrm{Mg}$ & ${ }^{49} \mathrm{Mn}-{ }^{49} \mathrm{Cr}$ & ${ }^{53} \mathrm{Fe}-{ }^{53} \mathrm{Co}$ & $\sigma_{\text {moy }}$ \\
\hline$\sigma_{B C S-n p}$ & 16.2 & 12.13 & 13.2 & 13.9 \\
\hline$\sigma_{S B C S-n p}$ & 1.79 & 3.37 & 0.22 & 1.79 \\
\hline$\sigma_{B C S}$ & 31.9 & 18.6 & 23.7 & 24.73 \\
\hline$\sigma_{S B C S}$ & 22.1 & 15.8 & 19.6 & 19.16 \\
\hline$\sigma_{[5]}$ & 10.4 & 3.2 & 20.1 & 11.23 \\
\hline$\sigma_{0}$ & 6.12 & 2.95 & 0.73 & 3.26 \\
\hline
\end{tabular}

\section{Results}

In all what follows, BCS and SBCS, respectively, refer to the method dealing with the pairing between like-particles, before and after the particle-number projection. BCS$\mathrm{np}$ and SBCS-np, respectively, correspond to their homologues in the isovector pairing case. We consider mirror nuclei $N \simeq Z$ since $n-p$ pairing effect is supposed to be maximal in these nuclei. We used single-particle energies of a deformed Woods-Saxon mean-field with the parameters described in Ref. [15] with a number of oscillator shells $N_{\max }=10$. The nuclear deformation is described by the elongation parameter $c$ and the neck parameter $h$ [15]. The pairing-strengths are determined such as to reproduce the "experimental" pairing gaps as described in Ref. [16].

\subsection{Isovector pairing effect}

We consider tree transitions, that is

$$
\begin{aligned}
& { }^{25} \mathrm{Al} \rightarrow{ }^{25} \mathrm{Mg}+e^{+}+v \\
& { }^{49} \mathrm{Mn} \rightarrow{ }^{49} \mathrm{Cr}+e^{+}+v \\
& { }^{53} \mathrm{Co} \rightarrow{ }^{53} \mathrm{Fe}+e^{+}+v
\end{aligned}
$$

The various parameters dealing with the six nuclei used in the present study are listed in Table 1 . The corresponding $\log f t$ values have been calculated using the BCS, SBCS, BCS-np and SBCS-np approaches (see Fig. 1). We have also added the values of $\log f t$ calculated using the simple approach used in Ref. [5]. They are compared with experimental data [17]. The relative discrepancy between the theoretical and experimental values [16] are summarized in Table 2. Surprisingly, in the pairing between likeparticles case, the BCS results obtained by means of the simple method described in Ref. [5] (the average value of the discrepancy is $11.23 \%$ ) are in better agreement with

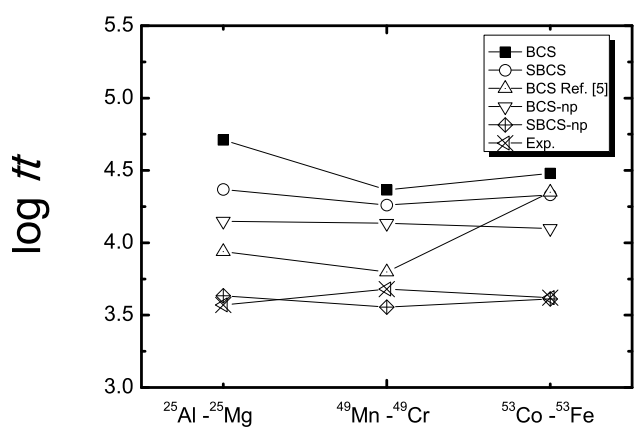

Figure 1. Comparison between the $\log f t$ values calculated within BCS, SBCS, BCS-np and SBCS-np approaches and experimental data. Values obtained using the simple BCS approach of Ref. [5] are also shown.

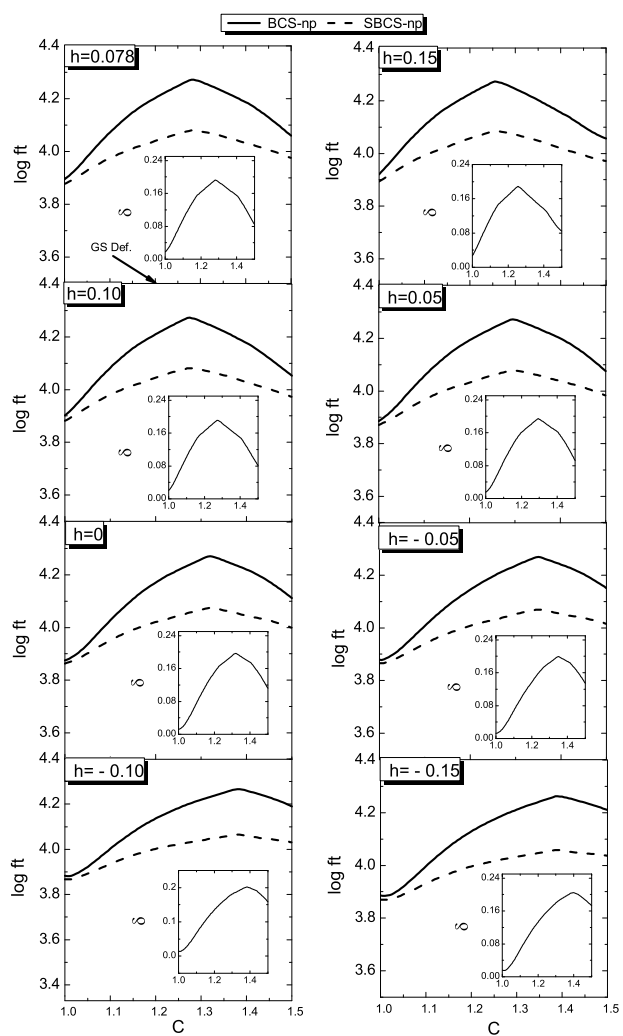

Figure 2. Variation of the $\log f t$ values as a function of $c$ for various values of $h$, within the BCS-np (solid lines) and SBCS$\mathrm{np}$ (dashed lines) approaches for the ${ }^{25} \mathrm{Al}-{ }^{25} \mathrm{Mg}$ transition. The value corresponding to the ground state of the mother nucleus is shown by an arrow in the upper left part. The daughter nucleus is spherical. $\delta$ is the difference between BCS-np and SBCS-np values.

experiment than those obtained using the effective singleparticle states, either before $(24.73 \%)$ or after the projection (19.16\%). The inclusion of the np pairing effects improves the results either before $(13.90 \%)$ or after the projection $(1.79 \%)$. However, just the results after the projection best reproduce experimental data. This fact shows, on 


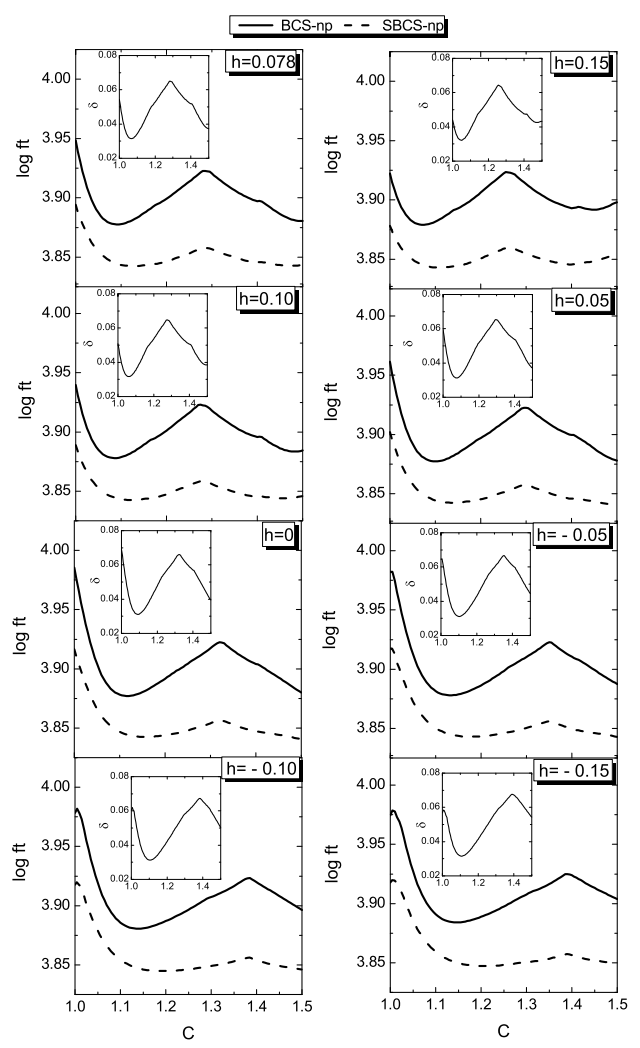

Figure 3. Same as Fig. 2 for $\left(c^{\prime}, h^{\prime}\right)=(1.1,0.1047)$ (ground state).

the one hand, the necessity of including the isovector pairing correlations and on the other hand, the necessity of the restoration of the particle-number conservation symmetry.

\subsection{Deformation effect}

We have then studied the effect of the deformation of the mother and daughter nuclei on the $\log f t$ values using the BCS-np and SBCS-np approaches. We considered the transition (13), chosen as an example. We denote the deformation parameters of the mother nucleus by $(c, h)$ and the daughter one by $\left(c^{\prime}, h^{\prime}\right)$. We have tried to change simultaneously the values of $(c, h)$ and $\left(c^{\prime}, h^{\prime}\right)$. To this end, we have plotted the variations of $\log f t$ as a function of $c$ for fixed values of $h$. In the present work, we consider the spherical state $\left(c^{\prime}=1, h^{\prime}=0\right)$ (see Fig. 2) and ground state $\left(c^{\prime}=1.1, h^{\prime}=0.1047\right)$ (see Fig. 3$)$ of the daughter nucleus. It seems from this set of results that: (i) a small variation of the deformation parameters of a nucleus, affects significantly the values of $\log f t$; (ii) it is not only important to include the isovector pairing when calculating the values of $\log f t$, but also to restore the symmetry of particle-number conservation broken under the BCS-np approach.

\section{Conclusion}

The particle-number projection effect, in the isovector pairing case, on the evaluation of the $\beta^{+}$transition probability amplitudes (of Fermi and Gamow-Teller types), and thus on $\log f t$, has been studied. With this aim, we used a discrete particle-number projection method. As a first step, we have established the expressions of the various beta transition probability amplitudes that strictly conserve the particle number. We considered some transitions, for nuclei with $N=Z \pm 1$, since the isovector pairing effect is assumed to be important in them using the Woods-Saxon mean field. The results of the present model are compared to those obtained before the projection and to those of the pairing between like-particles case (before and after the projection). It is shown, on the one hand, that it is necessary to include the isovector pairing correlations and on the other hand, that it is necessary to restore the particlenumber symmetry. Furthermore, the good agreement between the experimental data and the results of the present work indicates that couplings to the possible collective degrees of freedom (which have been omitted in the present study) play a minor role in the considered transitions. We also studied the effect of the deformation of the mother and daughter nuclei on the value of $\log f t$. The importance of right choice of the deformation parameters has been shown.

\section{References}

[1] S. Frauendorf and A.O. Macchiavelli, Prog. Part. Nucl. Phys. 78, 24 (2014)

[2] J. Engel et al., Phys. Rev. C 55, 1781 (1997)

[3] F. Frisk, Physica Scripta 57, 612 (1998)

[4] O. Moreno et al., Phys. Rev. C 73, 054302 (2006)

[5] J. Suhonen, From Nucleons to Nucleus (SpringerVerlag, Berlin, 2007)

[6] W. Satula and R. Wyss, Nucl. Phys. A 676, 120 (2000)

[7] K. Sieja and A. Baran, Acta Phys. Polonica B 37, 107 (2006)

[8] M. Fellah, T.F. Hammann, D.E. Medjadi, Phys. Rev. C 8, 1585 (1973)

[9] N.H. Allal et al., Eur. Phys. J. A 27, 301 (2006)

[10] S. Kerrouchi, D. Mokhtari, N.H. Allal, M. Fellah, Int. J. Mod. Phys. E 18, 141 (2009)

[11] S. Kerrouchi, N.H. Allal, M. Fellah, M. Douici, Int. J. Mod. Phys. E 19, 1383 (2010)

[12] S. Kerrouchi, N.H. Allal, M. Fellah, M.R. Oudih, Int. J. Mod. Phys. E 24, 1550014 (2015)

[13] J.C. Hardy et al., Nucl. Phys. A 509, 429 (1990)

[14] A. Bohr and B. Mottelson, Nuclear Structure, Vol. 1: Single-Particle Motion (Benjamin, New York, 1969)

[15] H.C. Pauli, Phys. Rep. 7, 35 (1973)

[16] F. Šimkovic et al., Phys. Rev. C 68, 054319 (2003)

[17] M.-M. Bé et al., Table of Radionuclides, ISBN 27272-0201-6 (CEA Saclay, 1999) 